hep-th/0209259

\title{
Noncommutativity Parameter and Composite Fermions
}

\author{
Ahmed Jellal * \\ Institut für Physik, Technische Universität Chemnitz, \\ D-09107 Chemnitz, Germany
}

\begin{abstract}
We determine some particular values of the noncommutativity parameter $\theta$ and show that the Murthy-Shankar approach is in fact a particular case of a more general one. Indeed, using the fractional quantum Hall effect (FQHE) experimental data, we give a measurement of $\theta$. This measurement can be obtained by considering some values of the filling factor $\nu$ and other ingredients, magnetic field $B$ and electron density $\rho$. Moreover, it is found that $\theta$ can be quantized either fractionally or integrally in terms of the magnetic length $l_{0}$ and the quantization is exactly what Murthy and Shankar formulated recently for the FQHE. On the other hand, we show that the mapping of the FQHE in terms of the composite fermion basis has a noncommutative geometry nature and therefore there is a more general way than the Murthy-Shankar method to do this mapping.
\end{abstract}

Keywords: Noncommutative geometry, fractional quantum Hall effect, composite fermions. PACS Nos.: 02.40.Gh, 73.43.-f, 71.10.Pm

${ }^{*}$ E-mail: jellal@gursey.gov.tr 


\section{Introduction}

Laughlin's wavefunctions [1]

$$
\Psi^{\mathrm{L}}=\prod_{i<j}\left(z_{i}-z_{j}\right)^{m} e^{-\frac{1}{4} \sum_{i}\left|z_{i}\right|^{2}}
$$

actually are good wavefunctions for describing the fractional quantum Hall effect (FQHE) [2, 3] at filling factor $\nu=\frac{1}{m}, m$ odd integer. However, the situation at most other filling factors is somewhat less clear. Several attempts are proposed to extend Laughlin's theory by adopting different approaches and assumptions. In particular, Jain [4, 5] introduced the composite fermion ( $\mathrm{CF}$ ) concepts. Indeed, Jain's idea is to explain the FQHE in terms of the integer quantum Hall effect (IQHE) by using the attached flux notion where each electron is assumed to be surrounded by an integer number of flux. Subsequently, by constructing a velocity operator in terms of the standard operator momentum and weakened vector potential, Murthy and Shankar [6, 7] proposed a Hamiltonian formalism for the FQHE mapped in terms of the CF degrees of freedom.

Recently with Dayi, we proposed [8] an approach based on noncommutative geometry tools [9] to describe the FQHE of a system of electrons. In fact, the corresponding filling factor is found to be

$$
\nu_{\mathrm{DJ}}=\frac{\pi}{2} \rho\left(l^{2}-\theta\right)
$$

which is identified with the observed fractional values $f=1 / 3,2 / 3,1 / 5, \cdots$. This approach also allowed us to make a link with the CF approach [4, 5] of the FQHE by setting an effective magnetic field

$$
B_{\mathrm{DJ}}=\frac{B}{1-\theta l^{2}}
$$

similar to that felt by the CF's.

In this paper we would like to return to our former work [8] in order to add some relevant applications. Indeed, by considering the experimental data of two different systems exhibiting the FQHE, we determine explicitly the corresponding values of the noncommutativity parameter $\theta$. Under some assumptions, we find that $\theta$ can be quantized in terms of the magnetic length and the quantization is nothing but what Murthy and Shankar defined when dealing with the FQHE in terms of the CF's. Moreover, we present a generalization of the Murthy-Shankar approach for the FQHE.

Section 2 is a review of the derivation of the Hall conductivity of a two dimensional system of electrons subject to an external magnetic field and living on both planes, commutative and 
noncommutative. These serve as a guide in section 3 in order to determine some particular values of $\theta$ and in the meantime quantize it. In section 4 after recalling briefly the MurthyShankar approach, we show that this approach has a noncommutative nature and therefore there is a more general approach.

\section{Hall conductivity}

In this section we shall review the determination of the Hall conductivity for a two dimensional system of electrons subject to a magnetic field $B$. In fact, we start by recalling the commutative case and end up with the noncommutative one.

\subsection{Commutative plane}

A system of an electron living on the plane $(x, y)$ and in the presence of an uniform external $B$ and $E$ fields can be described by the following Hamiltonian

$$
H=\frac{1}{2 m}\left[\left(p_{x}-\frac{e B}{2 c} y\right)^{2}+\left(p_{y}+\frac{e B}{2 c} x\right)^{2}\right]+e E x
$$

where the gauge is chosen to be symmetric $\vec{A}=\frac{B}{2}(-y, x)$ and the scalar potential is fixed to be $\phi=-x E_{x}$.

$H$ can be diagonalised simply by considering a couple of creation and annihilation operators. Then, let us define the first pair $[8$

$$
\begin{aligned}
& b^{\dagger}=-2 i p_{\bar{z}}+\frac{e B}{2 c} z+\lambda \\
& b=2 i p_{z}+\frac{e B}{2 c} \bar{z}+\lambda
\end{aligned}
$$

and also the second

$$
\begin{aligned}
& d=2 i p_{z}-\frac{e B}{2 c} \bar{z} \\
& d^{\dagger}=-2 i p_{\bar{z}}-\frac{e B}{2 c} z
\end{aligned}
$$

where $\lambda=\frac{m c E}{B}$ and $z=x+i y$ is the complex coordinate. These sets satisfy the commutation relations

$$
\begin{aligned}
& {\left[b, b^{\dagger}\right]=2 m \hbar \omega} \\
& {\left[d^{\dagger}, d\right]=2 m \hbar \omega}
\end{aligned}
$$

where $\omega=\frac{e B}{m c}$ is the cyclotron frequency. The other commutators vanish. By using the above operators, we can write $H$ as

$$
H=\frac{1}{4 m}\left(b^{\dagger} b+b b^{\dagger}\right)-\frac{\lambda}{2 m}\left(d^{\dagger}+d\right)-\frac{\lambda^{2}}{2 m} .
$$


From the eigenvalue equation

$$
H \Psi=E \Psi
$$

we obtain eigenstates and energy spectrum:

$$
\begin{aligned}
& \Psi_{(n, \alpha)} \equiv\left|n, \alpha>=\frac{1}{\sqrt{(2 m \hbar \omega)^{n} n !}} e^{i\left(\alpha y+\frac{m \omega}{2 \hbar} x y\right)}\left(b^{\dagger}\right)^{n}\right| 0> \\
& E_{(n, \alpha)}=\frac{\hbar \omega}{2}(2 n+1)-\frac{\hbar \lambda}{m} \alpha-\frac{\lambda^{2}}{2 m}
\end{aligned}
$$

where $n=0,1,2 \cdots$ and $\alpha \in \mathbb{R}$.

The corresponding Hall conductivity $\sigma_{\mathrm{H}}$ can be derived by using the definition of the related current operator $\vec{J}$, such as

$$
\vec{J}=-\frac{e \rho}{m}\left(\vec{p}+\frac{e}{c} \vec{A}\right)
$$

where $\rho$ is the electron density. Moreover, the expectation value of $\vec{J}$ can be calculated with respect to the eigenstates $\mid n, \alpha>$ (10). Therefore, we obtain

$$
\begin{aligned}
& <J_{x}>=0 \\
& <J_{y}>=-\left(\frac{\rho e c}{B}\right) E .
\end{aligned}
$$

The second equation implies that the Hall conductivity $\sigma_{\mathrm{H}}$ is

$$
\sigma_{\mathrm{H}}=-\frac{\rho e c}{B} .
$$

Using the definition of the filling factor:

$$
\nu=2 \pi \rho l_{0}^{2}
$$

where $l_{0}=\sqrt{\frac{\hbar c}{e B}}$ is the magnetic length, we can write $\sigma_{\mathrm{H}}$ as

$$
\sigma_{\mathrm{H}}=-\nu \frac{e^{2}}{h}
$$

\subsection{Noncommutative plane}

In this subsection, we review a generalization [8] of the last section in terms of noncommutative geometry [9]. Notations will be slightly changed in order to be coherent with our further analysis. In doing so, let us start by introducing the noncommutativity between the spatial coordinates, such as

$$
\left[x^{i}, x^{j}\right]=i \theta^{i j}
$$


where $\theta^{i j}=\epsilon^{i j} \theta$ is the noncommutativity parameter and $\epsilon^{12}=-\epsilon^{21}=1$. Basically, we are forced in this case to replace $f g(x)=f(x) g(x)$ by the relation

$$
f(x) \star g(x)=\left.\exp \left[\frac{i}{2} \theta^{i j} \partial_{x^{i}} \partial_{y^{j}}\right] f(x) g(y)\right|_{x=y}
$$

where $f$ and $g$ are two arbitrary functions, supposed to be infinitely differentiable. As a consequence, now we are going to deal with quantum mechanics by considering the following algebra

$$
\begin{aligned}
& {\left[x^{i}, x^{j}\right]=i \theta^{i j}} \\
& {\left[p^{i}, x^{j}\right]=-i \delta^{i j}} \\
& {\left[p^{i}, p^{j}\right]=0 .}
\end{aligned}
$$

Actually, we can write down the noncommutative version of the Hamiltonian (4). In doing so, let us notice that $H$ acts on an arbitrary function $\Psi(\vec{r}, t)$ as

$$
H \star \Psi(\vec{r}, t)=H^{\mathrm{nc}} \Psi(\vec{r}, t)
$$

which implies that $H^{\mathrm{nc}}$ is

$$
H^{\mathrm{nc}}=\frac{1}{2 m}\left[\left(\gamma p_{x}-\frac{e B}{2 c} y\right)^{2}+\left(\gamma p_{y}+\frac{e B}{2 c} x\right)^{2}\right]+e E\left(x-\frac{\theta}{2 \hbar} p_{y}\right)
$$

where $\gamma$ is a new parameter and defined to be $\gamma=1-\theta l^{-2}$ and $l=2 l_{0}$.

Now, one can use a similar process as in the previous section to diagonalise $H^{\text {nc }}$. Let us define the following operators

$$
\begin{aligned}
& \tilde{b}^{\dagger}=-2 i \gamma p_{\bar{z}}+\frac{e B}{2 c} z+\lambda_{-} \\
& \tilde{b}=2 i \gamma p_{z}+\frac{e B}{2 c} \bar{z}+\lambda_{-}
\end{aligned}
$$

and

$$
\begin{aligned}
& \tilde{d}=2 i \gamma p_{z}-\frac{e B}{2 c} \bar{z} \\
& \tilde{d}^{\dagger}=-2 i \gamma p_{\bar{z}}-\frac{e B}{2 c} z .
\end{aligned}
$$

The sets of operators $\left(\tilde{b}, \tilde{b}^{\dagger}\right)$ and $\left(\tilde{d}, \tilde{d}^{\dagger}\right)$ commute with each other. Moreover, they verify the commutation relations

$$
\begin{aligned}
& {\left[\tilde{b}, \tilde{b}^{\dagger}\right]=2 m \hbar \tilde{\omega}} \\
& {\left[\tilde{d}^{\dagger}, \tilde{d}\right]=2 m \hbar \tilde{\omega}}
\end{aligned}
$$

where $\tilde{\omega}$ and the $\lambda_{ \pm}$are given by

$$
\begin{aligned}
& \tilde{\omega}=\gamma \omega \\
& \lambda_{ \pm}=\lambda \pm \frac{e m E \theta}{4 \gamma \hbar} .
\end{aligned}
$$


To ensure these equations hold and for further analysis, we assume that the condition $\theta \neq l^{2}$ is satisfied. In terms of the above creation and annihilation operators, the Hamiltonian $H^{\text {nc }}$ takes the form

$$
H^{\mathrm{nc}}=\frac{1}{4 m}\left(\tilde{b}^{\dagger} \tilde{b}+\tilde{b} \tilde{b}^{\dagger}\right)-\frac{\lambda_{+}}{2 m}\left(\tilde{d}^{\dagger}+\tilde{d}\right)-\frac{\lambda_{-}^{2}}{2 m} .
$$

As before, we can solve the eigenvalue equation

$$
H^{\mathrm{nc}} \Psi^{\mathrm{nc}}=E^{\mathrm{nc}} \Psi^{\mathrm{nc}}
$$

to get the eigenstates:

$$
\Psi_{(n, \alpha, \theta)}^{\mathrm{nc}} \equiv\left|n, \alpha, \theta>=\frac{1}{\sqrt{(2 m \hbar \tilde{\omega})^{n} n !}} e^{i\left(\alpha y+\frac{m \tilde{\omega}}{2 \hbar} x y\right)}\left(\tilde{b}^{\dagger}\right)^{n}\right| 0>
$$

and the corresponding eigenvalues:

$$
E_{(n, \alpha, \theta)}^{\mathrm{nc}}=\frac{\hbar \tilde{\omega}}{2}(2 n+1)-\frac{\hbar \gamma \lambda_{+}}{m} \alpha-\frac{m}{2} \lambda_{-}^{2}
$$

where $n=0,1,2 \ldots$ and $\alpha \in \mathbb{R}$.

The conductivity resulting from the Hamiltonian $H^{\text {nc }}$ is determined by defining the current operator $\vec{J}^{\mathrm{nc}}$ on the noncommutative plane as

$$
\overrightarrow{J^{\mathrm{nc}}}=-\frac{e \rho \gamma}{m}\left(\gamma \vec{p}+\frac{e}{c} \vec{A}+\vec{a}\right)
$$

where the $\vec{a}$ vector is

$$
\vec{a}=\left(0,-\frac{m e E \theta}{2 \hbar \gamma}\right) .
$$

Its expectation value is calculated with respect to the eigenstates $\mid n, \alpha, \theta>(27)$ and is found to be

$$
\begin{aligned}
& <J_{x}^{\mathrm{nc}}>=0 \\
& <J_{y}^{\mathrm{nc}}>=-\left(\gamma \frac{\rho e c}{B}\right) E .
\end{aligned}
$$

Therefore, the Hall conductivity on the noncommutative plane of electrons, denoted by $\sigma_{\mathrm{H}}^{\mathrm{nc}}$, is

$$
\sigma_{\mathrm{H}}^{\mathrm{nc}}=-\gamma \frac{\rho e c}{B}
$$

and as before we can define an effective filling factor

$$
\nu_{\text {eff }}=\frac{\gamma \Phi_{0} \rho}{B}
$$

corresponding to an effective magnetic field:

$$
B_{\text {eff }}=\frac{B}{\gamma}
$$

where $\Phi_{0}=\frac{h c}{e}$ is the unit flux. To close this section, let us notice that the commutative analysis is recovered if the noncommutativity parameter $\theta$ is switched off. 


\section{Measurement and quantization of $\theta$}

Before we start, let us mention that in our work [8] we offered two interpretations for equation (32). In particular (32) can be seen as a result of the FQHE at fractional filling factor $f$. Identifying

$$
\left.\nu_{\mathrm{eff}}\right|_{\theta=\theta_{\mathrm{H}}}=f
$$

we find

$$
\theta_{\mathrm{H}}=\frac{2 \Phi_{0}}{\pi B}\left(1-f \frac{B}{\Phi_{0} \rho}\right)
$$

which tells us when $\theta$ is fixed to be $\theta_{\mathrm{H}}$, one can envisage the Hall effect on noncommutative plane as the usual fractional quantum Hall effect.

\subsection{Measurement}

Next, we determine explicit values of $\theta$ by using experimental observations. Such measurements are possible since we actually have a relation (36) governing the present parameters. Basically, to measure $\theta$ one can use experimental data where $f$ and the corresponding magnetic field are well-known. To do this task, we should fix the FQHE system and the corresponding ingredients. For instance, let us consider two different systems of electrons:

\section{GaAs/AlGaAs heterostructure:}

This system was the subject of many investigations dealing with the FQHE at low temperature and high mobility. In [10], the authors obtained some measurements by considering the present system of electron density $\rho=1.510^{11} \mathrm{~cm}^{-2}$. Their experimental data was reported as follows: The energy gap of the FQHE state at $\nu=4 / 3$ is $0.27 K$ at $B=7.3 T$, while it is $0.19 K$ at $B=5.9 T$ for $\nu=5 / 3$ state.

At this stage, we can have a fixed value of the noncommutativity parameter. Indeed, the magnetic length can be measured in terms of the magnetic field such as

$$
l_{0}[\mathrm{~m}]=25.6510^{-9}[B(T)]^{-1 / 2} .
$$

On the other hand, let us rewrite (36) as follows

$$
\theta_{\mathrm{H}}\left[\mathrm{m}^{2}\right]=4 l_{0}^{2}-\frac{2 f}{\pi \rho} .
$$


Now, we can have an explicit value of $\theta$ corresponding to the above experimental data. Therefore for the $\nu=4 / 3$ state, we obtain

$$
\left.\theta_{\mathrm{H}}\right|_{\nu=4 / 3}=0.205510^{-11} \mathrm{~cm}^{2}
$$

while the $\nu=5 / 3$ state leads us to have

$$
\left.\theta_{\mathrm{H}}\right|_{\nu=5 / 3}=0.261710^{-11} \mathrm{~cm}^{2}
$$

This is a way to give some hints on spatial noncommutativity. Moreover, another possibility is given in terms of Aharonov-Bohm effect, where an experiment is proposed to measure $\theta$ [ $]$.

\section{GaAs- $\mathrm{Al}_{0.3} \mathrm{Ga}_{0.7} \mathrm{As}$ heterostructure:}

Here we are going to give a table including some experimental results and the corresponding measurement of the noncommutativity parameter $\theta$. The above system is considered in [1] and their results are listed in the table. Therefore, from the author's observation and equation (38), one can end up with a table involving a summary of the values for the quantized Hall conductivity of the various FQHE and the corresponding values of $\theta$ :

\begin{tabular}{|c|c|c|c|c|c|}
\hline$\nu$ & $\rho\left(\mathrm{cm}^{-2}\right)$ & $B(\mathrm{kG})$ & $4 l_{0}^{2}\left(\mathrm{~cm}^{2}\right)$ & $\theta\left(\mathrm{cm}^{2}\right)$ & $\frac{\theta}{4 l_{0}^{2}}$ \\
\hline $1 / 3$ & $1.5310^{11}$ & 190 & $0.13851010^{-11}$ & $0.00025710^{-11}$ & 0.001855 \\
\hline $2 / 3$ & $2.4210^{11}$ & 150 & $0.17544610^{-11}$ & $0.00002010^{-11}$ & 0.000113 \\
\hline $2 / 5$ & $2.1310^{11}$ & 220 & $0.11962210^{-11}$ & $0.00000810^{-11}$ & 0.000066 \\
\hline $3 / 5$ & $2.1310^{11}$ & 147 & $0.17902610^{-11}$ & $0.00039310^{-11}$ & 0.002195 \\
\hline $5 / 3$ & $2.0610^{11}$ & 53 & $0.49654510^{-11}$ & $0.01878010^{-11}$ & 0.037821 \\
\hline $3 / 7$ & $2.1310^{11}$ & 206 & $0.12775110^{-11}$ & $0.00040510^{-11}$ & 0.003170 \\
\hline
\end{tabular}

From this table we observe that the ratio $\frac{\theta}{4 l_{0}^{2}}$ is very much smaller than one, which means that $\theta \ll 4 l_{0}^{2}$. Therefore, for this system the corresponding effective magnetic field can be approximated as

$$
B_{\mathrm{eff}} \approx B+\frac{B \theta}{4 l_{0}^{2}} .
$$




\subsection{Quantization}

Once the noncommutativity parameter $\theta$ is linked to the fractional filling factor (36), then one can ask about the quantization of $\theta$ in terms of the magnetic length $l_{0}$. To clarify this point, let us demand that $\sigma_{H}$ is nothing but referring to the IQHE, namely

$$
\sigma_{H}=-i \frac{e^{2}}{h}
$$

where $i$ is integer value. Then, (36) can be written as

$$
\theta_{\mathrm{H}}=4 l_{0}^{2}\left(1-\frac{f}{i}\right)
$$

this tells us that $\theta$ is actually quantized either fractionally or integrally. Now we would like to make contact with the Murthy-Shankar $c^{2}$ parameter [6], which is related to the CF theory. Indeed, let us consider the case where the filling factor $f$ is identified to the Jain series

$$
f=\frac{i}{2 i p+1}
$$

where $p=0,1, \cdots$. Now injecting (44) in (43), we find

$$
\theta_{\mathrm{H}}=4 l_{0}^{2}\left(\frac{2 i p}{2 i p+1}\right) .
$$

Setting $k=i p=0,1,2, \cdots$, we obtain

$$
\theta_{\mathrm{H}}=4 l_{0}^{2}\left(\frac{2 k}{2 k+1}\right) .
$$

Therefore the fractional value $\frac{2 k}{2 k+1}$ is exactly the quantity $c^{2}$ defined recently by Murthy and Shankar [6] to formulate a Hamiltonian for the FQHE in the CF basis. Then, we can write the above relation as

$$
\frac{\theta_{\mathrm{H}}}{4 l^{2}}=c^{2} .
$$

We will come back to the Murthy-Shankar method in the next section when we will talk about the CF's.

\section{Composite fermions}

In this section, we show that the recent results obtained by Murthy and Shankar concerning the CF's are particular cases of what is derived before [8] by considering electrons moving on 
the noncommutative plane. CF's are particles carrying an even number $2 p(p=1,2, \cdots)$ of flux quanta (vortices). They have the same charge, spin and statistics as the usual particles, but they differ from them since they experience an effective magnetic field

$$
B^{*}=B \pm 2 p \rho \Phi_{0}
$$

Before going on, we note that a system of electrons living on the noncommutative plane in the presence of an external magnetic field $B$ can be seen as a set of CF's subject to an effective magnetic field $B_{\text {eff }}$ and living on the usual plane. This statement is supported by the following relation 8

$$
\left.B_{\mathrm{eff}}\right|_{\theta=\theta_{\mathrm{c}}}=B^{*}
$$

where $B_{\text {eff }}$ is given in (34). This equation leads us to have

$$
\theta_{\mathrm{c}}=\frac{2 \Phi_{0}}{\pi B}\left[1 \pm\left(1-2 p \frac{\rho \Phi_{0}}{B}\right)^{-1}\right]
$$

\subsection{Murthy-Shankar approach}

In this subsection, we are going to review shortly the recent development of Murthy and Shankar 6] for the FQHE. Indeed, the authors considered a CF Hilbert space, where each fermion is described by a coordinate $\vec{r}$ and momentum $\vec{p}$, by constructing the following operator

$$
\vec{\pi}=\vec{p}+e \overrightarrow{A^{*}}
$$

where the weakened vector potential $A^{*}$

$$
A^{*}=\frac{A}{2 p s+1}
$$

is what the CF sees, where $p$ and $s$ are integers. In terms of these variables, the electron guiding center $\vec{R}_{e}$ takes the form

$$
\vec{R}_{e}=\vec{r}-\frac{l^{2}}{(1+c)} \hat{\vec{z}} \times \vec{\pi}
$$

where the c parameter is given by

$$
c^{2}=\frac{2 p s}{2 p s+1}
$$

It is easy to see that

$$
\left[R_{e x}, R_{e y}\right]=-i l_{0}^{2} .
$$


Actually, $\vec{R}_{e}$ can be written in terms of the CF guiding center and cyclotron coordinates $\vec{R}$ and $\vec{\eta}$, such that

$$
\vec{R}_{e}=\vec{R}+c \vec{\eta} .
$$

Another pair of guiding center-like coordinates commuting with $\vec{R}_{e}$ can be defined

$$
\vec{R}_{v}=\vec{R}+\frac{1}{c} \vec{\eta}
$$

which can also be mapped in terms of $\vec{r}$ and $\vec{\pi}$ :

$$
\begin{aligned}
& \vec{R}_{v}=\vec{r}+\frac{l^{2}}{c(1+c)} \hat{\vec{z}} \times \vec{\pi} \\
& {\left[R_{v x}, R_{v y}\right]=i l^{2} / c^{2} .}
\end{aligned}
$$

These correspond to the guiding center coordinates of a particle of charge $-c^{2}=-2 p s /(2 p s+1)$, which is precisely the charge of an object that must pair with the electron to form the CF called pseudo-vortex coordinate, since it has the same charge as a 2s-fold vortex in Laughlin states.

Since $\vec{R}_{v}$ has a magnetic algebra charge of $-c^{2}$, and there is one pseudo-vortex per electron, one can see that it is always at filling factor:

$$
\nu^{\prime}=\frac{\nu}{-c^{2}}=-\frac{1}{2 s}
$$

corresponding to the bosonic Laughlin wavefunctions [12]:

$$
\Psi^{L}=\prod_{i<j}\left(z_{i}-z_{j}\right)^{2 s} e^{-\sum_{i} \frac{c^{2}\left|z_{i}\right|^{2}}{4}}
$$

For many speculations about this approach and related matters, one can see the author's original work [6].

\subsection{Noncommutative nature}

We show that the Murthy-Shankar approach has a noncommutative nature and therefore there is a theory more general and is actually noncommutativity parameter $\theta$ dependent. In fact, we have seen in the beginning of this section that the CF theory can be envisaged as a particular theory of electrons moving on the $\mathrm{NC}$ plane and this statement is governed by equation (50). To process, let us write (150) as follows

$$
\frac{\theta_{\mathrm{c}}}{4 l_{0}^{2}}=\frac{4 p \pi \rho l_{0}^{2}}{4 p \pi \rho l_{0}^{2} \pm 1} .
$$


Remembering that the filling factor is given by $\nu=2 \pi \rho l_{0}^{2}$, putting this in relation (61), we find

$$
\frac{\theta_{\mathrm{c}}}{4 l^{2}}=\frac{2 p \nu}{2 p \nu \pm 1}
$$

In a similar way and for the same reason as we have seen in the last subsection, let us define a noncommutative filling factor as

$$
\nu_{\mathrm{nc}}^{\prime}=\frac{\nu^{*}}{-\theta_{\mathrm{c}} / 4 l^{2}}
$$

for any filling factor $\nu^{*}$ characterizing the quantum Hall effect. It is equivalent to

$$
\nu_{\mathrm{nc}}^{\prime}=-\nu^{*}\left(1 \pm \frac{1}{2 p \nu}\right)
$$

and the corresponding wavefunctions can be written as

$$
\Psi^{\mathrm{nc}}=\prod_{i<j}\left(z_{i}-z_{j}\right)^{1 /-\nu_{\mathrm{nc}}^{\prime}} e^{-\frac{\theta_{\mathrm{c}}}{16 l^{2}} \sum_{i}\left|z_{i}\right|^{2}} .
$$

This may be a general way to see that the Murthy-Shankar method is in fact a particular case of noncommutative analysis. To prove this statement, let us demand that $\nu$ is referred to the IQHE by fixing $\nu \equiv i=1,2, \cdots$. Therefore, we end up with

$$
\left.\frac{\theta_{\mathrm{c}}}{4 l^{2}}\right|_{\nu \equiv i}=\frac{2 i p}{2 i p \pm 1}
$$

showing that

$$
\left.\frac{\theta_{\mathrm{c}}}{4 l^{2}}\right|_{\nu^{*} \equiv i}=c^{2}
$$

Moreover, equation (64) becomes

$$
\left.\nu_{\mathrm{nc}}^{\prime}\right|_{\nu \equiv i}=-\nu^{*}\left(1 \pm \frac{1}{2 i p}\right)
$$

Fixing $\nu^{*}$ to be $\frac{i}{2 i p \pm 1}$, we obtain

$$
\left.\nu_{\mathrm{nc}}^{\prime}\right|_{\nu \equiv i} \equiv \nu^{\prime}=-\frac{1}{2 p}
$$

which is nothing but the Murthy-Shankar filling factor (59). Therefore we arrived to conclude that considering the weakened vector potential $A^{*}$ seen by CF's is equivalent to having a set of particles living on noncommutative space. Clearly, this analysis gives one example among other applications of noncommutative geometry in physics and shows how NC can serve to study some condensed matter physics phenomena. 


\section{Conclusion}

By exploring the experimental data of some fractional quantum Hall systems a measurement of the noncommutativity parameter $\theta$ is given. In fact, two different heterostructures: GaAs/AlGaAs and GaAs- $\mathrm{Al}_{0.3} \mathrm{Ga}_{0.7} \mathrm{As}$ are considered and showing different values of $\theta$. For the first system, we obtained the $\theta$ values corresponding to the filling factors $\frac{4}{3}$ and $\frac{5}{3}$. While for the second one several values are determined and a comparison with respect to the magnetic length was given, see table. This measurement gives some hint on spatial noncommutativity.

On the other hand, we developed an analysis in terms of noncommutative geometry to generalize the recent Murthy-Shankar proposal and also to prove that their proposal has in fact a noncommutative origin.

\section{Acknowledgments}

I would like to thank P. Bouwknegt for his kind invitation to participate in a workshop on Noncommutative Geometry and Fractional Quantum Hall Effect at the University of Adelaide (02-06 August 2002), and also for his warm hospitality during my stay at his Department of Physics and Mathematical Physics where the present work has been completed. I am grateful to Ö.F. Dayi for his helpful comment and P. Dawson for reading the manuscript.

\section{References}

[1] R.B. Laughlin Phys. Rev. B50 (1983) 1395.

[2] D.C. Tsui, H.L. Störmer and A.C. Gossard, Phys. Rev. Lett. 48 (1982) 1555.

[3] R.E. Prange and S.M. Girvin (editors), "The Quantum Hall Effect", (New York, Springer 1990).

[4] J.V. Jain, Phys. Rev. Lett. 63 (1989) 199; Phys. Rev. B41 (1990) 7653, Adv. Phys. 41 (1992) 105.

[5] O. Heinonen (editor), "Composite Fermions: A Unified View of Quantum Hall Regime", (World Scientific, 1998). 
[6] Ganpathy Murthy and R. Shankar, Hamiltonian Theory of the Fractional Quantum Hall Effect: Effect of Landau Level Mixing, cond-mat/0201082.

[7] Ganpathy Murthy and R. Shankar, Hamiltonian Theory of the FQHE, cond-mat/0205326.

[8] Ö.F. Dayi and A. Jellal, J. Math. Phys. 43 (2002) 4592.

[9] A. Connes, Noncommutative Geometry (Academic Press, London 1994).

[10] G.S. Boebinger, A.M. Chang, H.L. Stormer and D.C. Tsui, Phys. Rev. B32 (1985) 4268.

[11] A.M. Chang, B. Berglund, D.C. Tsui, H.L. Stormer and J.C.M. Hwang, Phys. Rev. Lett. 53 (1984) 997.

[12] D.H. Lee, Phys. Rev. Lett. 80 (1998) 4745. 